\title{
Swing-Reducing Flight Control System for an Underactuated Indoor Miniature Autonomous Blimp
}

\author{
Qiuyang Tao, Junkai Wang, Zheyuan Xu, Tony X. Lin, Ye Yuan and Fumin Zhang
}

\begin{abstract}
This paper presents the first swing stabilization control for indoor miniature autonomous blimps (MABs). Indoor MABs are safe to operate in close proximities to humans and can fly for multiple hours, but swing oscillation is commonly observed due to their underactuated design and unique aerodynamic shape. In this paper, we analyze the flight characteristics of indoor MABs, and describe the design of the swing-reducing flight control system in detail. Key mechatronic designs for swingstabilization control are also presented. Experimental results show that the proposed controller can keep the blimp travel at the desired velocity while effectively stabilizing the swing oscillation. The swing-reducing velocity controller is then expanded for station-keeping and waypoint navigation in $3 \mathrm{D}$ space.
\end{abstract}

Index Terms-Miniature autonomous blimp; Swing Oscillation; flight control system.

\section{INTRODUCTION}

A ERIAL robots have been pushing the boundary of indoor capabilities by demonstrating application success in tasks such as surveillance and inspection [1]-[4]. However, existing aerial platforms have not developed the smooth and safe indoor operations necessary for human interaction. Existing drones like quadcopters are still notoriously unsatisfactory in aspects including safety and endurance [5]-[8]. For example, mini drones usually exhibit sub-ten minute flight times [9], and require cages and netted enclosures for safe indoor operation.

We develop the Georgia-Tech Miniature Autonomous Blimp (GT-MAB), a lighter-than-air robot that can safely operate in close proximities to humans and can fly for multiple hours. The GT-MAB features a saucer-shaped design without tail fin, symmetrical holonomic actuation, and a low-latency off-board control scheme. The GT-MAB has ducted thrusters and is naturally cushioned, which makes the robot safe to fly indoors around humans, even when collisions occur. The blimp has a diameter of around 0.7 meters, which ensures the mobility in confined indoor space, and makes it one of the smallest autonomous blimps in the world.

Our initial approach to the autonomous flight of the GTMAB decomposes its movement into a set of motion primitives [10]. This decoupled modeling and control approach has successfully supported many applications of the GT-MAB

This work was supported by ONR grants N00014-19-1-2556 and N0001419-1-2266; AFOSR grant FA9550-19-1-0283; NSF grants CNS-1828678, S\&AS-1849228 and GCR-1934836; NRL grants N00173-17-1-G001 and N00173-19-P-1412; and NOAA grant NA16NOS0120028.

Q. Tao, J. Wang, Z. Xu, T. X. Lin and F. Zhang are with the School of Electrical and Computer Engineering, Georgia Institute of Technology, Atlanta, GA 30332 USA (e-mail: qiuyang@gatech.edu; wangjk@gatech.edu; charles.xu@gatech.edu; tlin339@gatech.edu; fumin@gatech.edu).

Y. Yuan is with the School of Automation, Huazhong University of Science and Technology, Wuhan 430074, China (e-mail: yye@ hust.edu.cn).

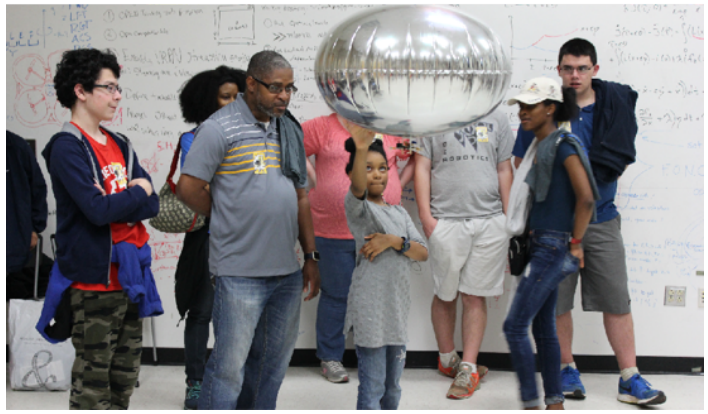

Fig. 1. Photo of the indoor miniature blimp, GT-MAB. The blimp can operate safely in close proximities to humans, and has endurance of multiple hours.

including 3D field mapping [10], [11], human-robot interaction (HRI) [12], [13], and testbed for deep-learning-based localization and multi-agent path planning [14], [15]. However, lateral and longitudinal oscillation is often observed due to the underactuated design and the unique aerodynamic shape. This undesirable oscillation could cause inaccurate sensor readings, unstable video streams from an onboard camera, and a less comfortable HRI experience. Our recent works [16], [17] identified the rotational motion of the GT-MAB and designed a control system to stabilize the swing oscillation during hovering flight. However, the control system developed in [17] cannot stabilize the oscillation in cruising flight.

The coupling between the translational and rotational movements and the non-linear dynamics are the major difficulties in stabilizing the swing oscillation. The GT-MAB has a unique aerodynamic design compared to outdoor airships. The outdoor airships have cigar-shaped envelopes and tail fins to stabilize and control their attitude. In contrast, the GT-MAB has a saucer-shaped envelope without tail fin. The saucer-shaped envelope and the elimination of fins provides a smoother outline for safe indoor operation. However, this configuration has very little damping to the swing oscillation. Also, without the control surfaces, the pitch and roll movements are highly coupled with the longitudinal and lateral movements.

Flight controllers for indoor blimps can be categorized into two groups based on whether a dynamic model is required. Due to the lack of dynamics model, large portion of the existing controller designs for indoor MABs are based on methods such as PID and fuzzy logic. Moreover, for the existing works in both categories, none of them has addressed the control of the coupled translational and rotational motion of indoor miniature blimps. The work [18] compared PID and fuzzy logic algorithms for altitude control, and presented a fuzzy logic controller for collision avoidance. Authors of the 
work [19] developed a biologically based flight controller with visual information from two camera inputs. PID controllers are designed in [20]-[22] for motion control and landing. Cooperative control of multiple neural networks are reported in [23] for the robustness with mechanical failures. The work [24] presented a behavior-based navigation system for an indoor blimp. The work [25] studied the control strategy under wind disturbance, and verified the proposed controller by simulation. Authors of [26] presented both modeling and controller design of a solar powered indoor blimp. The experimental results showed the blimp can successfully track a straight path in 3D space. [27] designed neuromorphic controllers, and trained the neural network using a simulator with identified dynamics model. The trained controller is then transferred on the physical blimp, and resulted very similar behavior with the simulated one. The works [28]-[30] designed a predictorbased controller to compensate for the system latency. Experimental results showed the controller can keep the blimp at the desired altitude. However, only the altitude-related modeling and control was considered in [28]-[30]. Our previous work [10] and the recent paper [8] decomposed the movement of the blimp into a set of motion primitives, and designed the waypoint controllers by combining the primitive movements. Similarly, the recent work [31] designed flight controllers for the decoupled vertical and planar motions of an indoor blimp. Moreover, PID controllers were implemented for the motion primitives of MABs with tilting [6], quadcopter-like [32], and flow-deflecting [33] actuator configurations. Our previous work [17] designed a control system to stabilize the swing oscillation during hovering flight. Unfortunately, the control system introduced in [17] cannot stabilize the oscillation in while the blimp is in translational flight. Our recent paper [34] identified the motion model of the coupled translational and rotational movements of the indoor blimp, GT-MAB. However, the controller design was not presented in [34].

In this article, we present the first realization of swing stabilization while simultaneously keeping the blimp traveling at the desired velocity. The proposed autopilot design addresses the difficulties in reducing the swing oscillation of indoor MABs including model non-linearity, and tightly coupled translational and rotational movements. We develop a nestedloop controller to separate the fast rotational motion with the slower translational movement. Feedforward components are added to both inner and outer loops to account for the model non-linearity. Key designs of the GT-MAB platform are also introduced that serve as a foundation for the swing stabilization control. The experimental results show that the GT-MAB can track the desired velocity while effectively stabilizes the swing oscillation. The swing-reducing flight controller is then expanded to waypoint navigation in 3D space.

The rest of this article is organized as follows: In Section II, we introduce the key designs of the GT-MAB for swing stabilization. In Section III, the motion model of the GT-MAB is explained for controller design. In Section IV, we present the development of the swing-reducing fight control system and demonstrate experimental results. In Section V, we draw conclusion and discuss future work.

\section{Key Designs FOR SWIng REDUCTION}

\section{A. Symmetric Holonomic Actuation}

Applications of indoor blimps prioritize multidirectional maneuverability and precise positioning. Holonomic mobility is a highly preferred feature for indoor MABs. With holonomic maneuverability, the blimp can move in any direction without steering, and can make sharp turns in place. Moreover, the blimp can keep an onboard directional sensor (such as a camera) pointed along a direction of interest regardless of translational movement.

The GT-MAB features a unique thruster configuration for symmetric holonomic actuation where only forward thrust is required for each motor. As demonstrated in Fig. 2, there are four horizontally-mounted thrusters on the gondola, forming an X-shaped configuration. As a result, symmetric actuation can be achieved for the planar movement, as illustrated in Fig. 3. Moreover, without the need for frequently switching the direction of rotation, motors usually have higher efficiency, longer lifespan, and faster response. The symmetric holonomic actuation also serves as a key enabling technology for the 3DOF model reduction, and the station-keeping and waypoint navigation functionalities discussed in this article.

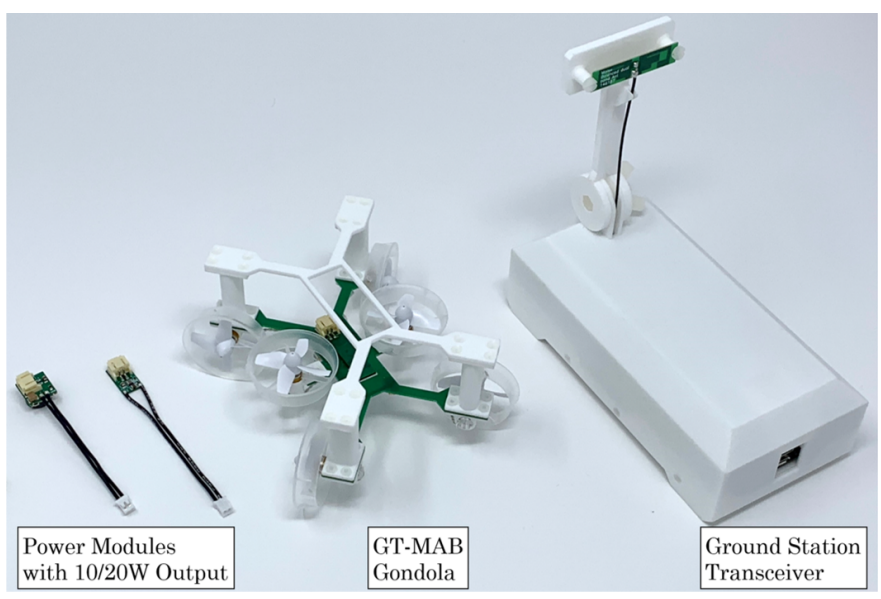

Fig. 2. Photo of the power modules (left), the gondola assembly (middle), and the ground station transceiver (right).

Indoor MABs usually do not equip shaft encoders on their thrusters due to limited payload. As a consequence, the actual propulsion varies as the battery becomes depleted. Therefore, as shown in Fig. 2, light-weight power modules are developed to provide constant voltage to the entire gondola despite the varying battery voltage. Step-down converters with high switching frequency are incorporated for high efficiency and reduced weight.

\section{B. Off-board Control with Reduced Latency}

The GT-MAB features a low-latency offboard control scheme, which allows computationally-intensive algorithms (such as computer vision) to be executed in real-time while simultaneously accommodates the strict weight and power limitations. However, as shown in Fig. 4, this system setup will introduce communication latency between the blimp and 


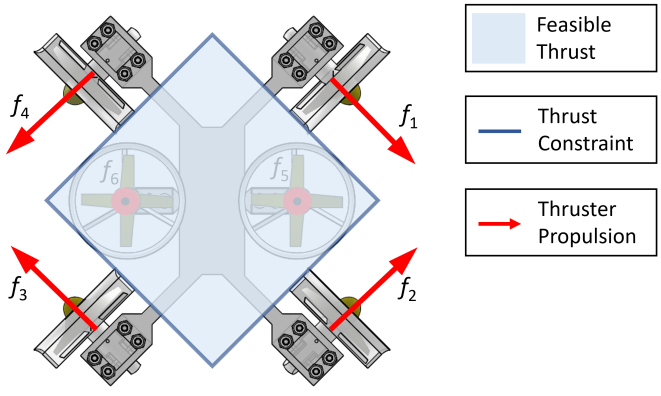

Fig. 3. Top view of the GT-MAB gondola. The horizontally mounted thrusters form an X-shaped configuration to achieve symmetric holonomic actuation.

the ground station. The control command computed by the ground station computer needs to be first sent to the blimp wirelessly before it can be executed by the onboard actuators. The impact of control system latency on indoor MABs was discussed in our previous work [17].

We develop a ground station transceiver to minimize the communication latency. The transceiver features $2 \mathrm{Mbps}$ onair data rate and a concise protocol for latency reduction. For instance, zero-order hold is implemented for unsuccessful package transactions instead of retransmission attempts. Shown in Fig. 5, it takes an average of 387 us to update the terminal voltages of the onboard thruters, after the control commands are received by the ground station transceiver. Moreover, the wireless communication has update rate up to $2000 \mathrm{~Hz}$. When operated at the maximum update rate with $8 \mathrm{dBm}$ transmission power, the overall power consumption of the gondola is less than $50 \mathrm{~mW}$ without motor action.

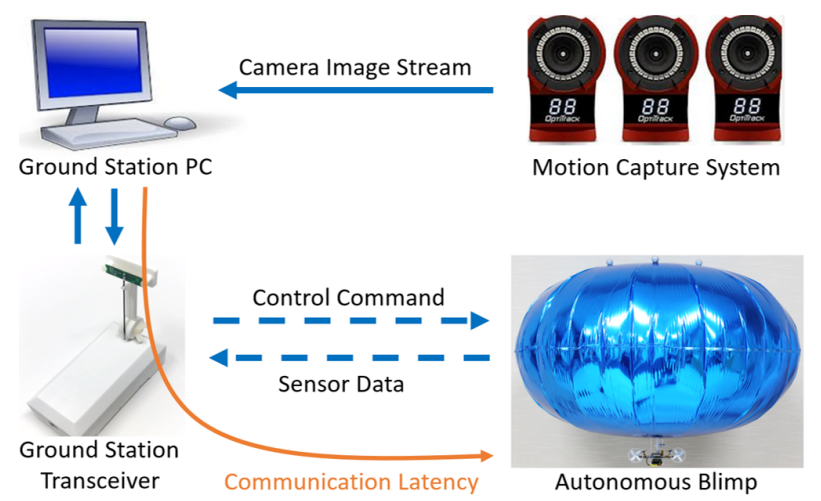

Fig. 4. System setup of GT-MAB. Blue solid arrows denote wired connection and blue dashed arrows represent wireless communication. The communication latency between the blimp and the ground station is annotated in orange.

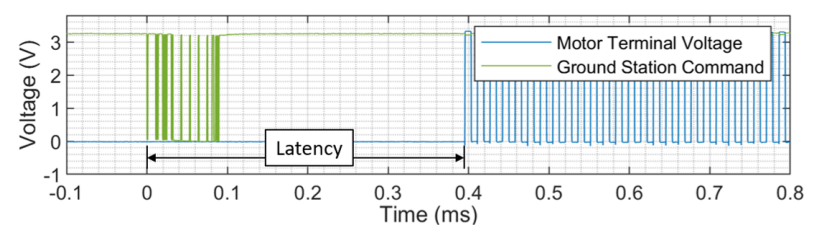

Fig. 5. Waveform of the control command signal received by the ground station transceiver and the corresponding motor terminal voltage.

\section{Motion Model of GT-MAB}

\section{A. Coordinate Frames and Kinematics}

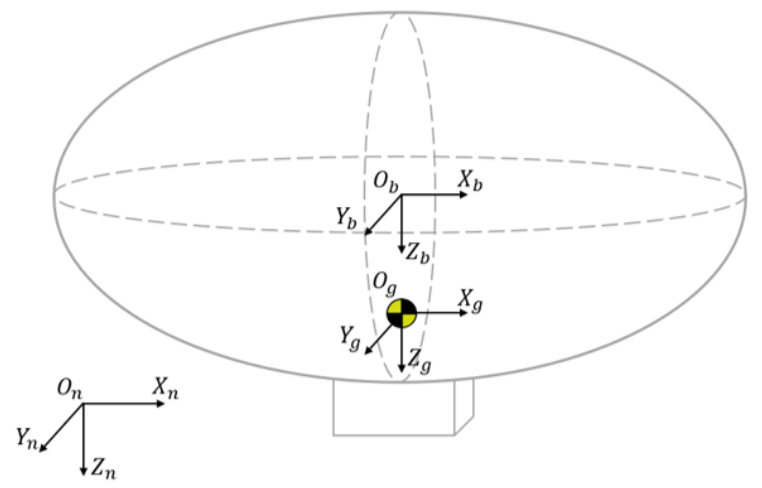

Fig. 6. Definition of the inertial and body frames of the GT-MAB.

Fig. 6 demonstrates the definition of the inertial frame $\{n\}$, and the body-fixed frames $\{b\}$ and $\{g\}$ that are separately attached at the center of buoyancy (CB) and the center of gravity (CG). It is more convenient to represent the motion model at $\mathrm{CB}$ due to the symmetrical properties of the envelope. Meanwhile, it is simpler to describe the movement of the blimp at CG for controller design. The pose of the GT-MAB is denoted as $\boldsymbol{\eta}_{b / n}^{n}=\left[\boldsymbol{p}_{b / n}^{n}, \boldsymbol{\Theta}_{n b}\right]^{\top}$, which stands for the pose of $\{b\}$ with respect to $\{n\}$ expressed in the inertial frame. $\boldsymbol{p}_{b / n}^{n} \in \mathbb{R}^{3}$ and $\Theta_{n b} \in \mathbb{S}^{3}$ represent the position and orientation separately. The instantaneous velocity of the blimp decomposed in the body frame $\{b\}$ is described by $\boldsymbol{\nu}_{b / n}^{b}=\left[\boldsymbol{v}_{b / n}^{b}, \boldsymbol{\omega}_{b / n}^{b}\right]^{\top}$, where $\boldsymbol{v}_{b / n}^{b} \in \mathbb{R}^{3}$ and $\boldsymbol{\omega}_{b / n}^{b} \in \mathbb{R}^{3}$ are the linear and angular velocities. From [35], the relationship between $\boldsymbol{\nu}_{b / n}^{b}$ and the rate of change of $\eta_{b / n}^{n}$ is given by:

$$
\dot{\boldsymbol{\eta}}_{b / n}^{n}=\left[\begin{array}{c}
\dot{\boldsymbol{p}}_{b / n}^{n} \\
\dot{\boldsymbol{\Theta}}_{n b}
\end{array}\right]=\left[\begin{array}{cc}
\boldsymbol{R}_{b}^{n}\left(\boldsymbol{\Theta}_{n b}\right) & \boldsymbol{0}_{3 \times 3} \\
\mathbf{0}_{3 \times 3} & \boldsymbol{T}_{\boldsymbol{\Theta}}\left(\boldsymbol{\Theta}_{n b}\right)
\end{array}\right]\left[\begin{array}{c}
\boldsymbol{v}_{b / n}^{b} \\
\boldsymbol{\omega}_{b / n}^{b}
\end{array}\right],
$$

where $\boldsymbol{R}_{b}^{n}\left(\boldsymbol{\Theta}_{n b}\right)$ and $\boldsymbol{T}_{\boldsymbol{\Theta}}\left(\boldsymbol{\Theta}_{n b}\right)$ are the rotation and transformation matrices. $\Theta_{n b}=[\phi, \theta, \psi]^{\top}$, and $\phi, \theta, \psi$ are the roll, pitch and yaw angles of the blimp separately.

\section{B. 3-DOF Motion Model at CG}

The GT-MAB has symmetric design around the vertical axes and capable of pointing the thrust vector in arbitrary 3D direction. Therefore, our previous work [34] has reduced the full 6-DOF motion model of the GT-MAB to 3-DOF movement on a vertical plane. Shown in Fig. 7, if the blimp starts from rest at position A, and targets at waypoint B, the ideal trajectory can be contained within a vertical plane that includes both points. The motion model of the coupled translational and rotational movement is recalled in Eq. (2), and the identification of the model parameters is presented in our previous works [16], [34]. 


$$
\begin{aligned}
& \left(m_{R B}+m_{A x}\right) \dot{v}_{x, b / n}^{b}+m_{R B} r_{z, g / b}^{b} \dot{\omega}_{y, b / n}^{b} \\
& +\left(m_{R B}+m_{A z}\right) v_{z, b / n}^{b} \omega_{y, b / n}^{b}+D_{v x}^{C B} v_{x, b / n}^{b}=f_{x}^{b} \\
& \left(m_{R B}+m_{A z}\right) \dot{v}_{z, b / n}^{b}-m_{R B} r_{z, g / b}^{b}\left(\omega_{y, b / n}^{b}\right)^{2} \\
& -\left(m_{R B}+m_{A x}\right) v_{x, b / n}^{b} \omega_{y, b / n}^{b}+D_{v z}^{C B} v_{z, b / n}^{b}=f_{z}^{b} \\
& I_{y} \dot{\omega}_{y, b / n}^{b}+m_{R B} r_{z, g / b}^{b}\left(\dot{v}_{x, b / n}^{b}+v_{z, b / n}^{b} \omega_{y, b / n}^{b}\right)+D_{\omega y}^{C B} \omega_{y, b / n}^{b} \\
& +\left(m_{A x}-m_{A z}\right) v_{x, b / n}^{b} v_{z, b / n}^{b}+r_{z, g / b}^{b} m_{R B} \sin (\theta)=\tau_{y}^{b},
\end{aligned}
$$

where $m_{R B}, m_{A x}$ and $m_{A z}$ represent the overall rigid-body mass of the GT-MAB, and the added mass for the forward and vertical movements separately. $I_{y}$ denotes the total rotational inertia evaluated at CB. The pitch angle of the blimp and the angular rate are defined as $\theta$ and $\omega_{y, b / n}^{b}$. Similarly, $v_{x, b / n}^{b}$ and $v_{z, b / n}^{b}$ denotes the surge and heave velocities of CB expressed in the body frame $\{b\} . D_{v x}^{C B}, D_{v z}^{C B}, D_{\omega y}^{C B}, f_{x}^{b}, f_{x}^{b}$ and $\tau_{y}^{b}$ are the air drag coefficients and motor actuation along $X_{b}, Z_{b}$ and around $Y_{b}$ axes. $r_{z, g / b}^{b}$ is the distance between CB and CG.

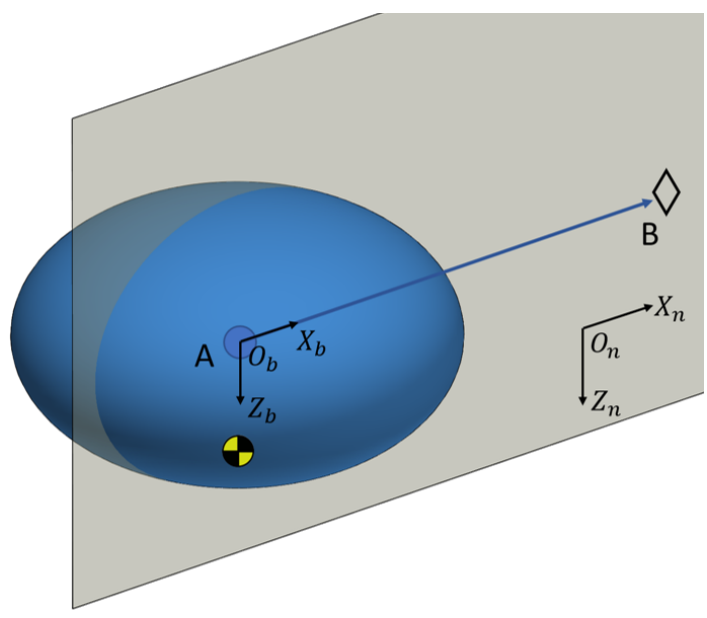

Fig. 7. Motion of the GT-MAB reduced to 3-DOF on a vertical plane.

Due to the bottom-heavy design of indoor MABs, we assume $\mathrm{CG}$ is the pivot of the swing oscillation. Fig. 8 demonstrates the position of both CB and CG while the blimp accelerates forward from rest. From the plot, the CB position will first move toward the opposite direction of the motor thrust, and then toward the same direction. In contrast, the CG position always moves toward the same direction as the thrust force. Therefore, we use the CG position to describe the motion of the GT-MAB for controller design. The motion model in Eq. (2) can be expressed at CG as:

$$
\begin{aligned}
& \left(m_{R B}+m_{A x}\right) \dot{\nu}_{x, g / n}^{b}-m_{A x} r_{z, g / b}^{b} \dot{\omega}_{y, g / n}^{b}+D_{v x}^{C B} v_{x, g / n}^{b} \\
& +\left(m_{R B}+m_{A z}\right) v_{z, g / n}^{b} \omega_{y, g / n}^{b}-D_{v x}^{C B} \omega_{y, g / n}^{b} r_{z, g / b}^{b}=f_{x}^{g} \\
& \left(m_{R B}+m_{A z}\right) \dot{v}_{z, g / n}^{b}+m_{A x} r_{z, g / b}^{b}\left(\omega_{y, g / n}^{b}\right)^{2} \\
& +D_{v z}^{C B} v_{z, g / n}^{b}-\left(m_{R B}+m_{A x}\right) v_{x, g / n}^{b} \omega_{y, g / n}^{b}=f_{z}^{g} \\
& I_{y}^{C G} \dot{\omega}_{y, g / n}^{b}-m_{A x} v_{z, g / n}^{b} \omega_{y, g / n}^{b} r_{z, g / b}^{b}-D_{v x}^{C B} r_{z, g / b}^{b} v_{x, g / n}^{b} \\
& +\left(m_{A x}-m_{A z}\right) v_{x, g / n}^{b} v_{z, g / n}^{b}+\left(D_{v x}^{C B} r_{z, g / b}^{b}{ }^{2}+D_{\omega y}^{C B}\right) \omega_{y, g / n}^{b} \\
& -m_{A x} r_{z, g / b}^{b} \dot{v}_{x, g / n}^{b}+g m_{R B} r_{z, g / b}^{b} \sin (\theta)=\tau_{y}^{g},
\end{aligned}
$$

where $v_{x, g / n}^{b}$ and $v_{z, g / n}^{b}$ represent the surge and heave velocities of CG. Since $\{b\}$ and $\{g\}$ are parallel, we get $\omega_{y, g / n}^{b}=\omega_{y, b / n}^{b}$. $f_{x}^{g}, f_{x}^{g}$ and $\tau_{y}^{g}$ are the actuation along $X_{g}, Z_{g}$ and around $Y_{g}$ axes. Similarly, $I_{y}^{C G}$ denotes the total rotational inertia at CG.

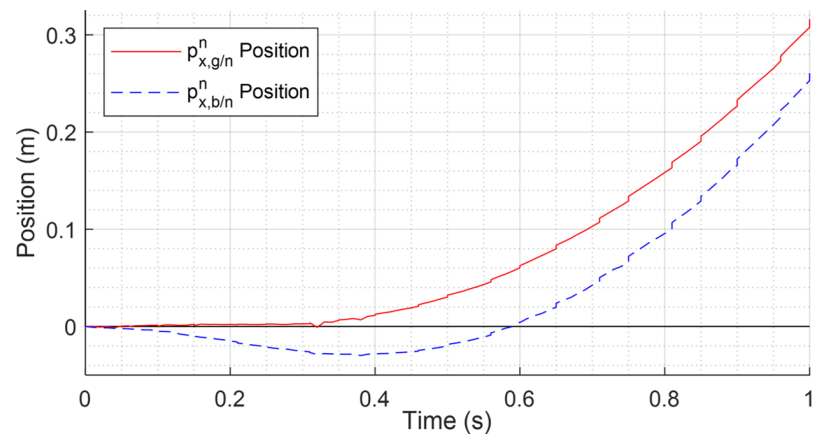

Fig. 8. Positions of CB and CG while the GT-MAB is accelerating forward along $X_{n}$ direction from rest. The CG always moves toward the same direction as that of the motor propulsion force.

\section{Motion Model Reduction}

The heave motion has neglectable coupling with the surge and pitch movements. Thus, we divide the 3-DOF motion into two groups of movements and design controllers separately.

1) Planar motion: For this motion primitive, we assume the blimp flies horizontally with zero vertical movement, namely $v_{z, g / n}^{b}=\dot{v}_{z, g / n}^{b}=0$. Therefore, the model of the planar motion can be simplified from Eq. (3) as:

$$
\begin{aligned}
& \dot{v}_{x, g / n}^{b}=\frac{D_{v x}^{C B} m_{A x} r_{z, g / b}^{b}{ }^{2}-D_{v x}^{C B} I_{y}^{C G}}{-m_{A x}{ }^{2} r_{z, g / b}^{b}{ }^{2}+I_{y}^{C G}\left(m_{R B}+m_{A x}\right)} v_{x, g / n}^{b} \\
& -\frac{g m_{A x} m_{R B} r_{z, g / b}^{b}{ }^{2}}{-m_{A x}{ }^{2} r_{z, g / b}^{b}{ }^{2}+I_{y}^{C G}\left(m_{R B}+m_{A x}\right)} \sin (\theta) \\
& +\frac{D_{v x}^{C B} I_{y}^{C G} r_{z, g / b}^{b}-D_{v x}^{C B} m_{A x} r_{z, g / b}^{b}{ }^{3}-D_{\omega y}^{C B} m_{A x} r_{z, g / b}^{b}}{-m_{A x}{ }^{2} r_{z, g / b}^{b}{ }^{2}+I_{y}^{C G}\left(m_{R B}+m_{A x}\right)} \omega_{y, g / n}^{b} \\
& +\frac{I_{y}^{C G} m_{A x} r_{z, g / b}^{b} r_{z, t / g}^{b}}{-m_{A x}{ }^{2} r_{z, g / b}^{b}{ }^{2}+I_{y}^{C G}\left(m_{R B}+m_{A x}\right)} f_{x}^{g} \\
& \dot{\theta}=\omega_{y, g / n}^{b} \\
& \dot{\omega}_{y, g / n}^{b}=\frac{D_{v x}^{C B} m_{R B} r_{z, g / b}^{b}}{-m_{A x}{ }^{2} r_{z, g / b}^{b}{ }^{2}+I_{y}^{C G}\left(m_{R B}+m_{A x}\right)} v_{x, g / n}^{b} \\
& -\frac{g\left(m_{R B}+m_{A x}\right) m_{R B} r_{z, g / b}^{b}}{-m_{A x}{ }^{2} r_{z, g / b}^{b}{ }^{2}+I_{y}^{C G}\left(m_{R B}+m_{A x}\right)} \sin (\boldsymbol{\theta}) \\
& -\frac{D_{\omega y}^{C B}\left(m_{R B}+m_{A x}\right)+D_{v x}^{C B} m_{R B} r_{z, g / b}^{b}{ }^{2}}{-m_{A x}{ }^{2} r_{z, g / b}^{b}{ }^{2}+I_{y}^{C G}\left(m_{R B}+m_{A x}\right)} \omega_{y, g / n}^{b} \\
& +\frac{m_{A x} r_{z, g / b}^{b}+\left(m_{R B}+m_{A x}\right)}{-m_{A x}{ }^{2} r_{z, g / b}^{b}+I_{y}^{C G}\left(m_{R B}+m_{A x}\right)} \tau_{y}^{g} \text {. }
\end{aligned}
$$


2) Vertical motion: For this motion primitive, we assume the heave motion is the dominant movement. Therefore, the model for the vertical motion can be found from Eq. (3) as:

$$
\left(m_{R B}+m_{A z}\right) \dot{v}_{z, g / n}^{b}+D_{v z}^{C B} v_{z, g / n}^{b}=f_{z}^{g} .
$$

The model in Eq. (5) is defined in the body frame, and needs to be converted to the inertial frame for altitude tracking. Given that the roll and pitch angles are small under the swingreducing fight controller, the vertical motion of the GT-MAB in the inertial frame can be approximated as:

$$
\left[\begin{array}{c}
\dot{p}_{z, g / n}^{n} \\
\ddot{p}_{z, g / n}^{n}
\end{array}\right]=\left[\begin{array}{cc}
0 & 1 \\
0 & \frac{-D_{v z}^{C B}}{m_{R B}+m_{A z}}
\end{array}\right]\left[\begin{array}{c}
p_{z, g / n}^{n} \\
\dot{p}_{z, g / n}^{n}
\end{array}\right]+\left[\begin{array}{c}
0 \\
\frac{1}{m_{R B}+m_{A z}}
\end{array}\right] f_{z}^{g} .
$$

The vertical motion model in Eq. (6) has a simple linear form, but the challenge is the asymmetrical actuation due to the thruster-hull interaction. Gain-switching and model predictive controllers are developed in our previous works [10], [36] to address this difficulty.

\section{Swing-Reducing Controller Design}

As described by Eq. (4), the planar motion of the GTMAB is non-linear and has strong couplings between the translational and rotational movements. To overcome these two difficulties, we design a nested-loop controller with feedforward and feedback components on both loops, as demonstrated in Fig. 9. The nested-loop design decouples the fast rotational motion from the slow translational movement, and utilizes the feedforward components of each loop to handle the non-linear dynamics.

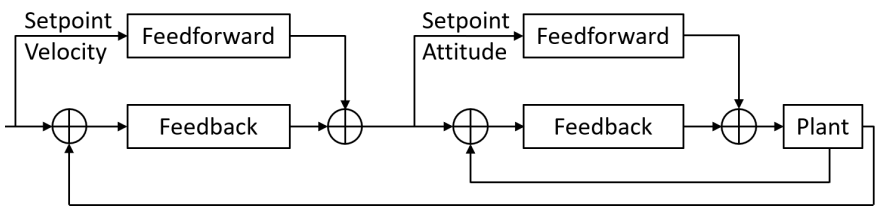

Fig. 9. Block diagram of the nested-loop controller with feedforward terms.

\section{A. Operating Point Analysis}

Given the non-linearity of the planar motion, we linearize the system around its equilibrium. At the operating point shown in Fig. 10, the blimp flies at a constant velocity $v_{x, g / n, 0}^{b}$, and the motors are generating a constant thrust $f_{x, 0}^{g}$ to compensate for the air drag. However, due to an offset between the force axes generated by the actuation and the air drag, an undesirable torque is generated that pitches the blimp up. This pitch-up torque is then compensated by the restoring torque from the buoyancy and gravitational forces. As a result, there exists a pitch angle $\theta_{0}$ while the blimp is flying at a given steady-state velocity. Therefore, the relationship between the system states at the operating point can be found as:

$$
\begin{gathered}
f_{x, 0}^{g}=r_{z, g / b}^{b} / r_{z, t / b}^{b} m_{R B} g \sin \left(\theta_{0}\right) \\
v_{x, g / n, 0}^{b}=\frac{r_{z, g / b}^{b} m_{R B} g}{D_{v x}^{C B} r_{z, t / b}^{b}} \sin \left(\theta_{0}\right),
\end{gathered}
$$

where $r_{z, t / b}^{b}$ is the vertical distance between the thrusters and the center of gravity.

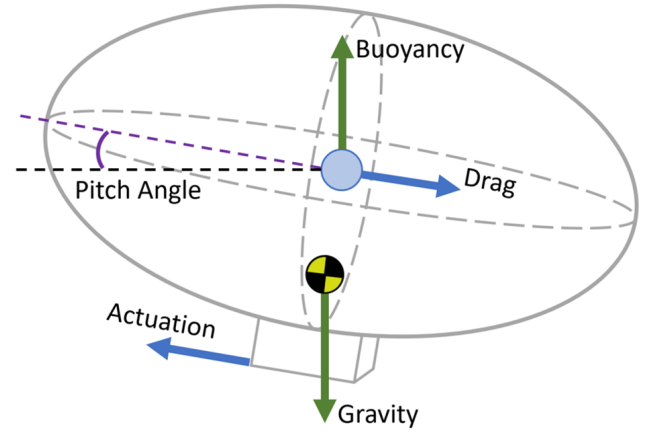

Fig. 10. Free body diagram of the GT-MAB at operating point.

\section{B. Nested-loop Controller Design}

The nested-loop design separates the coupling between the fast rotational motion from the slow translational movement. As demonstrated in Fig. 9, the inner loop tracks the attitude of the blimp for swing stabilization, while the outer loop maintains the desired velocity by adjusting the setpoint attitude for the inner loop. Here we summarize the four operating scenarios of the nested-loop controller:

- No disturbance: The actuation is solely from the feedforward terms to keep the blimp at the operating point.

- Disturbance in attitude: The outer loop remains the same as the equilibrium. The feedback portion of the inner loop compensates for the angular error.

- Disturbance in velocity: Feedback portion of the outer loop adjusts the setpoint attitude for the inner loop to reduce the velocity error.

- Disturbance in both attitude and velocity: The inner loop will first converge to the setpoint attitude due to faster dynamics. Then the outer loop will track the velocity.

1) Inner loop: For inner loop design, we assume the translational velocity is constant at the equilibrium, namely $v_{x, g / n}^{b}=v_{x, g / n, 0}^{b}$, given that adjusting the attitude of GT-MAB is significantly faster than changing its velocity. Therefore, by linearizing the blimp dynamics in Eq. (4) around the operating point, the motion model for attitude tracking can be found as:

$$
\begin{aligned}
\Delta \dot{\omega}_{y, g / n}^{b}= & -\frac{g\left(m_{R B}+m_{A x}\right) m_{R B} r_{z, g / b}^{b}}{-m_{A x}{ }^{2} r_{z, g / b}^{b}{ }^{2}+I_{y}^{C G}\left(m_{R B}+m_{A x}\right)} \Delta \theta \\
- & \frac{D_{\omega y}^{C B}\left(m_{R B}+m_{A x}\right)+D_{v x}^{C B} m_{R B} r_{z, g / b}^{b}{ }^{2}}{-m_{A x}{ }^{2} r_{z, g / b}^{b}{ }^{2}+I_{y}^{C G}\left(m_{R B}+m_{A x}\right)} \Delta \omega_{y, g / n}^{b} \\
+ & \frac{m_{A x} r_{z, g / b}^{b}+\left(m_{R B}+m_{A x}\right) r_{z, t / g}^{b}}{-m_{A x}{ }^{2} r_{z, g / b}^{b}{ }^{2}+I_{y}^{C G}\left(m_{R B}+m_{A x}\right)} \Delta f_{x}^{g},
\end{aligned}
$$

where the deviations from the operating point are defined as:

$$
\left[\Delta \theta, \Delta \omega_{y, g / n}^{b}, \Delta f_{x}^{g}\right]^{\top}=\left[\theta-\theta_{0}, \omega_{y, g / n}^{b}-\omega_{y, g / n, 0}^{b}, f_{x}^{g}-f_{x, 0}^{g}\right]^{\top} \text {. }
$$

Next, we design a state feedback controller to keep the pitch angle at the desired operating point, and the angular velocity to zero for swing stabilization:

$$
\Delta f_{x}^{g}=-k_{\theta} \Delta \theta-k_{\omega} \Delta \omega_{y, g / n}^{b},
$$


where $k_{\theta}$ and $k_{\omega}$ are the controller gains for the pitch angle error and the angular rate error. Therefore, the overall control effort of inner loop is summarized as:

$$
\begin{aligned}
f_{x}^{g} & =f_{x, 0}^{g}+\Delta f_{x}^{g} \\
& =r_{z, g / b}^{b} / r_{z, t / b}^{b} m_{R B} g \sin \left(\theta_{0}\right)-k_{\theta} \Delta \theta-k_{\omega} \Delta \omega_{y, g / n}^{b} .
\end{aligned}
$$

2) Outer loop: From the relationship between the equilibrium states in Eq. (7), the forward velocity of the blimp will converge to the desired value $v_{x, g / n, 0}^{b}$ by solely keeping the attitude at $\theta_{0}$. However, this open-loop process for velocity control is slow and may have noticeable error. For instance, the relationship in Eq. (7) may deviate since the CG position of the blimp will be drifted by the installation of payloads. Moreover, airflow is inevitable even in indoor environments. The difference between the airspeed and the ground speed may also change the relationship between the velocity and the attitude at the operating point.

Therefore, the outer loop controller is designed to adjust the operating point of the blimp for faster velocity tracking and compensate for the constant error. We assume there is no angular velocity given that the blimp flies stably with the swing-reducing inner loop controller. Therefore, by linearizing the blimp dynamics in Eq. (4) around the operating point, the motion model for velocity tracking can be found as:

$$
\begin{aligned}
\Delta \dot{v}_{x, g / n}^{b} & =-\frac{D_{v x}^{C B} r_{z, g / b}^{b}+D_{v x}^{C B} r_{z, t / g}^{b}}{r_{z, t / g}^{b}\left(m_{R B}+m_{A x}\right)+r_{z, g / b}^{b} m_{A x}} \Delta v_{x, g / n}^{b} \\
& +\frac{g m_{R B} r_{z, g / b}^{b}}{r_{z, t / g}^{b}\left(m_{R B}+m_{A x}\right)+r_{z, g / b}^{b} m_{A x}} \Delta \theta,
\end{aligned}
$$

where $\Delta v_{x, g / n}^{b}=v_{x, g / n}^{b}-v_{x, g / n, 0}^{b}$ is the velocity deviation from the equilibrium point. The motion model in Eq. (12) is a first order system with $\Delta \theta$ as input. To track the desired speed and eliminate steady-state error, we design a proportional-integral (PI) controller as the feedback portion of the outer loop:

$$
\theta_{\text {feedback }}=-k_{p} \Delta v_{x, g / n}^{b}-k_{i} \int_{0}^{t} \Delta v_{x, g / n}^{b} d t^{\prime},
$$

where $k_{p}$ and $k_{i}$ are the PI gains separately. Therefore, the setpoint attitude for the inner loop is summarized as:

$$
\begin{aligned}
& \theta_{\text {setpoint }}=\theta_{\text {feedforward }}+\theta_{\text {feedback }} \\
& =\arcsin \left(\frac{D_{v x}^{C B} r_{z, t / b}^{b}}{r_{z, g / b}^{b} m_{R B} g} v_{x, g / n, 0}^{b}\right)-k_{p} \Delta v_{x, g / n}^{b}-k_{i} \int_{0}^{t} \Delta v_{x, g / n}^{b} d t^{\prime} .
\end{aligned}
$$

\section{Experimental Results}

A series of experiments were designed to validate the proposed swing-reducing velocity controller. Test flights with setpoint velocities $0.05 \mathrm{~m} / \mathrm{s}, 0.1 \mathrm{~m} / \mathrm{s}, 0.15 \mathrm{~m} / \mathrm{s}, 0.2 \mathrm{~m} / \mathrm{s}, 0.25 \mathrm{~m} / \mathrm{s}$, and $0.3 \mathrm{~m} / \mathrm{s}$ were successfully conducted. Video demonstration of the experiments can be seen at https://youtu.be/3dOebPNpnNI.

Fig. 11 demonstrates the velocity and the pitch angle with $0.1 \mathrm{~m} / \mathrm{s}$ setpoint velocity. The blimp started from rest and accelerated to the goal velocity within two seconds. During the acceleration phase, the blimp was flying at a larger pitch angle for faster velocity tracking. The peak pitch angle during acceleration was approximately three times of that during cruising flight. During the cruising flight after the setpoint was reached, there were only small adjustments on the pitch angle to maintain the desired velocity.
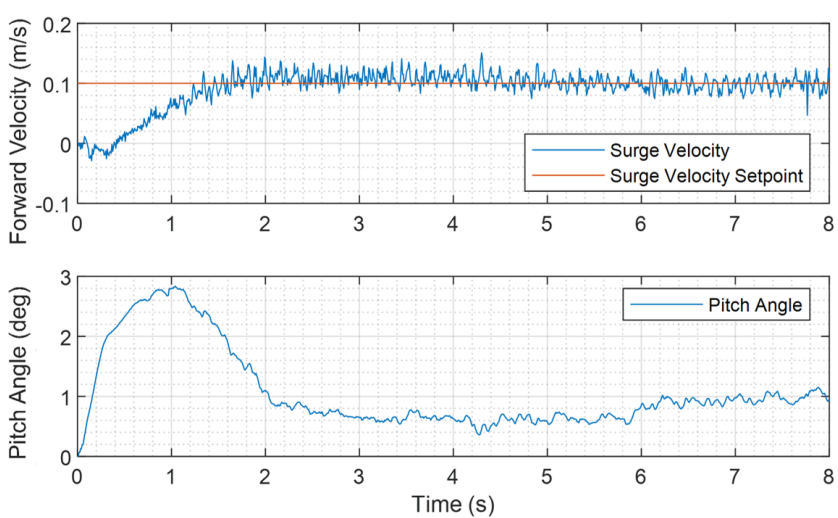

Fig. 11. Forward velocity and pitch angle of the GT-MAB during velocity tracking control. The blimp can accelerate to the setpoint velocity within two seconds, and can maintain the desired velocity.

The proposed controller design was also validated with added disturbance. As shown in Fig. 12, after the blimp reached the setpoint velocity, disturbance in both velocity and pitch angle was added by pulling the envelope of the blimp by hand. As seen in the plot, the blimp can recover from the disturbance within two seconds. Video demonstration of this experiment is available at https://youtu.be/b6KxRN2wAQs.
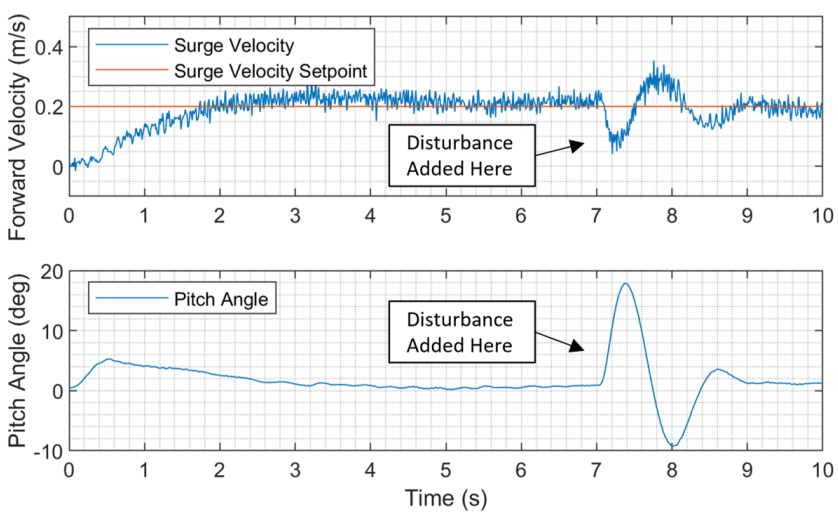

Fig. 12. Forward velocity control with added disturbance. The blimp can recover quickly from the added disturbance.

\section{Expansion to Waypoint Navigation}

The station-keeping and waypoint navigation functionalities are developed by expanding the swing-reducing velocity controller with position feedback. The swing-reducing velocity controller is first applied to both longitudinal and lateral movements. Then, position tracking is added in an outer loop that provides the setpoints for the velocity controllers. The position tracking is combined with heading and altitude control to navigate the blimp to the desired $3 \mathrm{D}$ position. Next, we implement a waypoint switching logic, which allows the GTMAB to automatically follow a sequence of waypoints. 
1) Velocity control for both surge and sway movements: Owing to the symmetrical design and holonomic actuation of the GT-MAB, the swing-reducing velocity controller is applied to both longitudinal and lateral movements. Thus the surge and sway velocities can be independently controlled for holonomic maneuverability.

A series of experiments were conducted to validate the simultaneous velocity control of both longitudinal and lateral motion. Fig. 13 demonstrates the velocity tracking with setpoints of surge and sway movements are both $0.2 \mathrm{~m} / \mathrm{s}$. The experiment was repeated by flying the blimp along different directions. Specifically, four directions forward and backward along body axes, and four additional orientations $45^{\circ}$ from the body axes. Video demonstration of the flight tests with all eight directions are available at https://youtu.be/Us-kmLRmYLY.
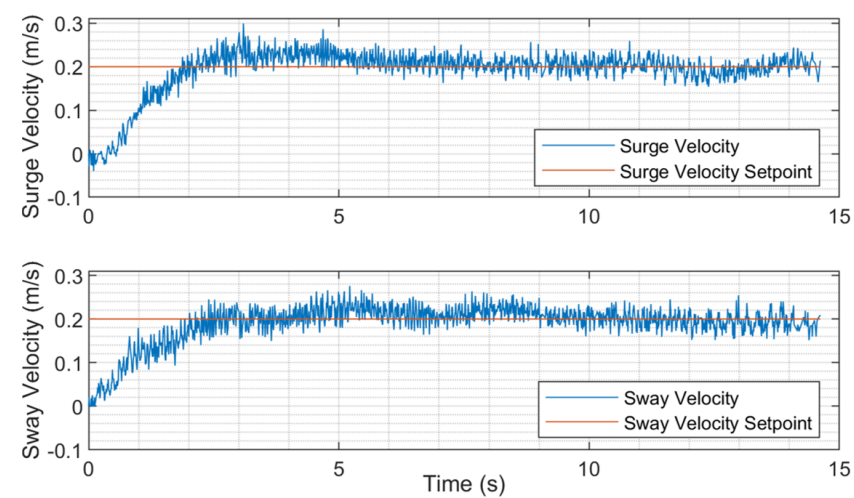

Fig. 13. Simultaneous velocity control of both surge and sway movements Both longitudinal and lateral motions can track the setpoint velocities.

2) Position tracking: The position tracking controller is designed to minimize the distance error to the desired location in the inertial frame. In this article, we focus on the planar position tracking which serves as an outer loop that provides the setpoints for the underlying velocity controllers. The altitude and heading control can be seen from our previous works [10], [17], [36].

With the feedback control for the distance errors, the setpoint velocities can be calculated as:

$$
\begin{aligned}
v_{x, g / n, \text { set point }}^{n} & =k_{x}^{n} e_{p x, g / n}^{n} \\
v_{y, g / n, \text { set point }}^{n} & =k_{y}^{n} e_{p y, g / n}^{n},
\end{aligned}
$$

where $e_{p x, g / n}^{n}$ and $e_{p y, g / n}^{n}$ are the distance errors along $X_{n}$ and $Y_{n}$ axes. $k_{x}^{n}$ and $k_{y}^{n}$ are the controller gains. For the GT$\mathrm{MAB}$, we let $k_{x}^{n}=k_{y}^{n}$ due to its symmetric design. With the kinematics described by Eq. (1), the setpoint velocities can then be decomposed to the body frame of the blimp.

Station-keeping flight was conducted with the position tracking controller. During the experiment, the proposed controller kept the blimp at the desired position despite of environmental disturbance. As listed in Table I, the standard deviation of the position drift was less than $6 \mathrm{~mm}$, and that of the attitude swing was below 0.15 degrees. Video demonstration of the flight test is available at https://youtu.be/uzpX-T3xFrY.
TABLE I

Position and AtTitude VARIATIONS of Station-KeEPing Flight

\begin{tabular}{|l|l|l|l|}
\hline Std. Dev. $p_{x, g / n}^{n}$ & Std. Dev. $p_{y, g / n}^{n}$ & Std. Dev. $\theta$ & Std. Dev. $\phi$ \\
\hline $5.9 \mathrm{~mm}$ & $5.5 \mathrm{~mm}$ & $0.13 \mathrm{deg}$ & $0.14 \mathrm{deg}$ \\
\hline
\end{tabular}

3) Waypoint navigation: A waypoint switching logic is implemented on top of the position tracking controller to navigate the blimp through a set of waypoints. Once the blimp comes within a specified distance to a waypoint, the blimp will then navigate toward the next waypoint in the sequence.

An experiment was designed to demonstrate the waypoint navigation with swing stabilization. As shown in Fig. 14, The blimp took off at the origin, and visited waypoints with planar positions of $(1.2,1.2),(-1,1.2),(-1,-1.2)$, and $(1.2,-$ 1.2 ), and heights of $0,0.2,0.4,0.6,0.8$ and 1.0 meters. Despite that roll and pitch angles are required for acceleration, deceleration and maintain the velocity, the standard deviation of these angles were only 0.85 and 1.02 degrees separately. Video demonstration of the flight test is available at https://youtu.be/c6TQtLOomgU.

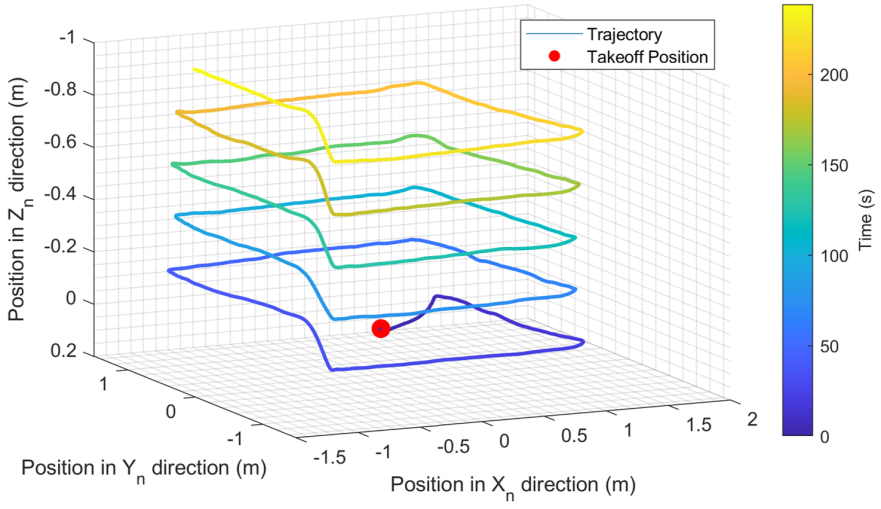

Fig. 14. 3D trajectory of the waypoint navigation experiment.

\section{CONCLUSIONS AND FUTURE WORKS}

This paper presents the first swing oscillation reduction among indoor miniature blimps. The translational and rotational movements of indoor MABs are nonlinear and coupled due to the underactuated design and unique aerodynamic shape. We develop a nested-loop controller with feedforward components to separate the coupled movements and account for the nonlinearity. Experimental results show that the flight controller can effectively reduce the swing oscillation while simultaneously keep the blimp cruising at the desired velocity. Key mechatronic designs are also presented which serve as a foundation for the swing stabilization control. The swingreducing flight controller is then expanded for station-keeping and waypoint navigation in 3D space.

We plan to refine the design of the GT-MAB for full 6DOF maneuverability. The fully-actuated MAB will allow the generation of force and torque in all three dimensions, which enables the tracking of arbitrary 6-DOF trajectories and expands the feasible flight maneuvers. The dynamics model and control system of the GT-MAB will also be extended to full 6-DOF in addition to the hardware design. 


\section{REFERENCES}

[1] S. Grzonka, G. Grisetti, and W. Burgard, "A fully autonomous indoor quadrotor," IEEE Transactions on Robotics, vol. 28, no. 1, pp. 90-100, Feb 2012.

[2] S. Shen, N. Michael, and V. Kumar, "Autonomous multi-floor indoor navigation with a computationally constrained micro aerial vehicle," in 2011 IEEE International Conference on Robotics and Automation, May 2011, pp. 2968-2969.

[3] V. Mai, M. Kamel, M. Krebs, A. Schaffner, D. Meier, L. Paull, and R. Siegwart, "Local positioning system using uwb range measurements for an unmanned blimp," IEEE Robotics and Automation Letters, vol. 3, no. 4, pp. 2971-2978, 2018.

[4] D. Palossi, A. Gomez, S. Draskovic, A. Marongiu, L. Thiele, and L. Benini, "Extending the lifetime of nano-blimps via dynamic motor control," Journal of Signal Processing Systems, vol. 91, no. 3-4, pp. 339-361, 2018.

[5] K. Asadi, A. K. Suresh, A. Ender, S. Gotad, S. Maniyar, S. Anand, M. Noghabaei, K. Han, E. Lobaton, and T. Wu, "An integrated UGVUAV system for construction site data collection," Automation in Construction, vol. 112, p. 103068, 2020.

[6] S. U. Ferdous, A. Mohammadi, and S. Lakshmanan, "Developing a low-cost autonomous blimp with a reduced number of actuators," in Unmanned Systems Technology XXI. SPIE 11021, 2019, pp. 73-80.

[7] W. Yamada, H. Manabe, and D. Ikeda, "Zerone: Safety drone with bladefree propulsion," in Proceedings of the 2019 CHI Conference on Human Factors in Computing Systems, 2019, pp. 1-8.

[8] V. Srisamosorn, N. Kuwahara, A. Yamashita, T. Ogata, S. Shirafuji, and J. Ota, "Indoor human face following with environmental fisheye cameras and blimp," Advanced Robotics, vol. 34, pp. 621-636, 2020.

[9] "Crazyflie 2.1 product page," https://store.bitcraze.io/products/crazyflie2-1, [Accessed: 3- Dec- 2020].

[10] S. Cho, V. Mishra, Q. Tao, P. Varnell, M. King-Smith, A. Muni, W. Smallwood, and F. Zhang, "Autopilot design for a class of miniature autonomous blimps," in Proc. of IEEE Conference on Control Technology and Applications (CCTA), 2017, pp. 841-846.

[11] Q. Tao, M. King-Smith, A. Muni, V. Mishra, S. Cho, P. Varnell, and F. Zhang, "Control theory - autonomous blimp," in IEEE CSS Video Clip Contest, 2015.

[12] N. Yao, E. Anaya, Q. Tao, S. Cho, H. Zheng, and F. Zhang, "Monocular vision-based human following on miniature robotic blimp," in IEEE International Conference on Robotics and Automation (ICRA), 2017, pp. 3244-3249.

[13] N. Yao, Q. Tao, W. Liu, Z. Liu, Y. Tian, P. Wang, T. Li, and F. Zhang, "Autonomous flying blimp interaction with human in an indoor space," Frontiers of Information Technology \& Electronic Engineering, vol. 20, no. 1, pp. 45-59, Jan 2019.

[14] L. Seguin, J. Zheng, A. Li, Q. Tao, and F. Zhang, "A deep learning approach to localization for navigation on a miniature autonomous blimp," in The 16 th IEEE International Conference on Control \& Automation, in press.

[15] J. Gibson, T. Schuler, L. McGuire, D. M. Lofaro, and D. Sofge, "Swarm and multi-agent time-based A* path planning for lta3 systems," Unmanned Systems, vol. 8, no. 3, pp. 253-260, 2020.

[16] Q. Tao, J. Cha, M. Hou, and F. Zhang, "Parameter identification of blimp dynamics through swinging motion," in 2018 15th International Conference on Control, Automation, Robotics and Vision (ICARCV), Nov 2018, pp. 1186-1191.

[17] Q. Tao, T. J. Tan, J. Cha, Y. Yuan, and F. Zhang, "Modeling and control of swing oscillation of underactuated indoor miniature autonomous blimps," Unmanned Systems, in press.

[18] P. Gonzalez, W. Burgard, R. Sanz, and J. L. Fernandez, "Developing a low-cost autonomous indoor blimp," Journal of Physical Agents, pp. 43-52, 2009.

[19] S. B. i Badia, P. Pyk, and P. F. M. J. Verschure, "A biologically based flight control system for a blimp-based uav," in Proceedings of the 2005 IEEE International Conference on Robotics and Automation, April 2005, pp. 3053-3059.

[20] T. Takaya, H. Kawamura, Y. Minagawa, M. Yamamoto, and A. Ohuchi, "PID landing orbit motion controller for an indoor blimp robot," Artificial Life and Robotics, vol. 10, no. 2, pp. 177-184, Nov 2006

[21] T. Takaya, H. Kawamura, Y. Minagawa, M. Yamamoto, and A. Ouchi, "Motion control in three dimensional round system of blimp robot," in 2006 SICE-ICASE International Joint Conference, Oct 2006, pp. 12911294.
[22] H. Kawamura, M. Yamamoto, T. Takaya, and A. Ohuchi, "Learning landing control of an indoor blimp robot for self-energy recharging," Artificial Life and Robotics, vol. 12, no. 1, pp. 116-121, Mar 2008

[23] H. Kawamura, H. Iizuka, T. Takaya, and A. Ohuchi, "Cooperative control of multiple neural networks for an indoor blimp robot," Artificial Life and Robotics, vol. 13, no. 2, pp. 504-507, Mar 2009.

[24] M. Mahn and M. Kemper, "A behaviour-based navigation system for an autonomous indoor blimp," IFAC Proceedings Volumes, vol. 39, no. 16, pp. 837-842, 2006, 4th IFAC Symposium on Mechatronic Systems.

[25] F. Hayato and S. Akira, "Wind-disturbance-based control approach for blimp robots," Electronics and Communications in Japan, vol. 97, no. 2, pp. 52-59, 2014.

[26] C. Wan, N. Kingry, and R. Dai, "Design and autonomous control of a solar-power blimp," in 2018 AIAA Guidance, Navigation, and Control Conference, AIAA SciTech Forum, 2018, p. 1588.

[27] J.-C. Zufferey, A. Guanella, A. Beyeler, and D. Floreano, "Flying over the reality gap: From simulated to real indoor airships," Autonomous Robots, vol. 21, no. 3, pp. 243-254, Nov 2006.

[28] Y. Wang, G. Zheng, D. Efimov, and W. Perruquetti, "Altitude control for an indoor blimp robot," IFAC-PapersOnLine, vol. 50, no. 1, pp. 15990 $15995,2017$.

[29] Y. Wang, G. Zheng, D. Efimov, and W. Perruquetti, "Improved altitude control method with disturbance compensation for an indoor blimp robot," in 2017 IEEE 56th Annual Conference on Decision and Control (CDC), 2017, pp. 3902-3907.

[30] Y. Wang, G. Zheng, D. Efimov, and W. Perruquetti, "Differentiator application in altitude control for an indoor blimp robot," International Journal of Control, vol. 91, no. 9, pp. 2121-2130, 2018.

[31] _ - "Disturbance compensation based controller for an indoor blimp robot," Robotics and Autonomous Systems, vol. 124, p. 103402, 2020.

[32] K. Watanabe, N. Okamura, and I. Nagai, "Closed-loop control experiments for a blimp robot consisting of four-divided envelopes," in IECON 2015 - 41st Annual Conference of the IEEE Industrial Electronics Society, 2015, pp. 2568-2573.

[33] S. H. Song, G. Y. Yeon, H. W. Shon, and H. R. Choi, "Design and control of soft unmanned aerial vehicle 's-cloud'," IEEE/ASME Transactions on Mechatronics, pp. 1-1, 2020.

[34] Q. Tao, M. Hou, and F. Zhang, "Modeling and identification of coupled translational and rotational motion of underactuated indoor miniature autonomous blimps," in 2020 16th International Conference on Control, Automation, Robotics and Vision (ICARCV), in press.

[35] T. I. Fossen, Handbook of Marine Craft Hydrodynamics and Motion Control. United Kingdom: John Wiley \& Sons, 2011.

[36] Q. Tao, "Design and control of an indoor miniature autonomous blimp," Ph.D. dissertation, Georgia Institute of Technology, Atlanta, GA, USA, 2020 , in press. 


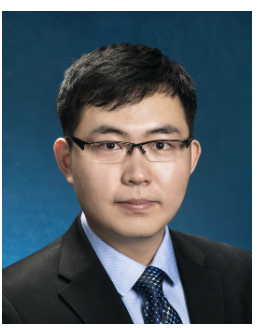

Qiuyang Tao received the B.S. in Electrical Engineering from the Georgia Institute of Technology, Atlanta GA, USA, in 2015. He is continuing his $\mathrm{Ph} . D$. studies in Electrical and Computer Engineering at the Georgia Institute of Technology. Qiuyang's current research interests include the design, modeling and control of novel robotic and mechatronic systems.

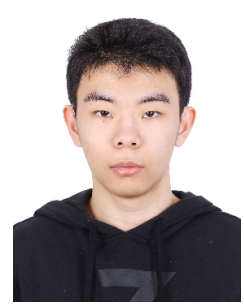

Junkai Wang received the B.S. in Automation from Beihang University, Beijing, China, in 2019. He is continuing his M.S. studies in Electrical and Computer Engineering at the Georgia Institute of Technology. Junkai's current research interets include the analysis and design of robotic control systems.

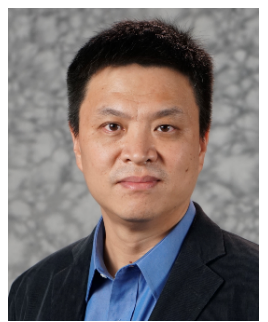

Fumin Zhang received the B.S. and M.S. degrees from Tsinghua University, Beijing, China, in 1995 and 1998, respectively, and the Ph.D. degree from the Department of Electrical and Computer Engineering, University of Maryland, College Park, in 2004. He joined the School of ECE, Georgia Institute of Technology in 2007, where he is a Professor. He was a Lecturer and Postdoctoral Research Associate in the Mechanical and Aerospace Engineering Department, Princeton University from 2004 to 2007. His research interests include marine autonomy, mobile sensor networks, and theoretical foundations for battery supported cyber-physical systems. He received the NSF CAREER Award in 2009, and the ONR YIP Award in 2010.

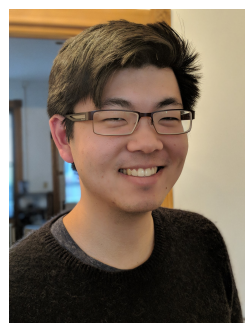

Zheyuan Xu received the B.S. in Electrical Engineering and Computer Science from Georgia Institute of Technology, Atlanta GA, USA, in 2020. $\mathrm{He}$ is continuing his M.S. studies in University of Washington, located at Seattle, WA. Zheyuan's current research interests include machine learning on cyber-physical systems.

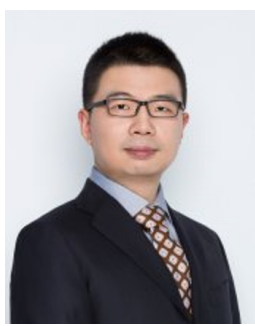

Ye Yuan received the B.Eng. degree (Valedictorian) from the Department of Automation, Shanghai Jiao Tong University, Shanghai, China, in 2008, and the M.Phil. and Ph.D. degrees from the Department of Engineering, University of Cambridge, Cambridge, U.K., in 2009 and 2012, respectively. He has been a Full Professor at the Huazhong University of Science and Technology, Wuhan, China since 2016. Prior to this, he was a Postdoctoral Researcher at UC Berkeley, a Junior Research Fellow at Darwin College, University of Cambridge. His research interests include system identification and control with applications to cyber-physical systems. Dr. Yuan has received the China National Recruitment Program of 1000 Talented Young Scholars, the Dorothy Hodgkin Postgraduate Awards, Microsoft Research Ph.D. Scholarship, Best of the Best Paper Award at the IEEE Power and Energy Society General Meeting. 